\title{
Les villes moyennes périphériques à l'épreuve des tendances économiques et démographiques. L'exemple de Saguenay
}

\author{
Martin Simard ${ }^{\mathrm{a}}$
}

RÉSUMÉ. Malgré son poids démographique non négligeable, Saguenay constitue une région métropolitaine de recensement (RMR) plutôt modeste parmi les 33 RMR canadiennes. De plus, à l'instar de Sudbury ou de Thunder Bay, Saguenay est localisée en périphérie des grands corridors urbano-industriels et sa population stagne ou décline au gré des recensements. Dans ce contexte, quel est l'avenir des villes moyennes situées aux marges de l'écoumène, comme Saguenay? Comment accueillir des immigrants dans un environnement social homogène? Faut-il appréhender un clivage progressif des besoins et des opportunités entre les villes moyennes de l'arrière-pays et les grandes métropoles? Nous tenterons de répondre à ces diverses interrogations.

\begin{abstract}
$\boldsymbol{A B S T R A C T}$. Despite its significant demographic importance, the Saguenay region is a rather small census metropolitan area (CMA) among the 33 Canadian CMAs. Like Sudbury or Thunder Bay, Saguenay is located at the periphery of major urban-industrial corridors and its population stagnates or declines from one census to another. In this context, what is the future of the midsized cities located at the edge of the ecumene, such as the Saguenay region? How do you take in immigrants in a generally homogeneous social environment? Will there be a gradual divide between the midsized cities of the hinterland and the large metropolitan areas? We will attempt to address these important issues.
\end{abstract}

Saguenay est une région métropolitaine de recensement (RMR) de 160000 habitants. Selon cette catégorisation de Statistique Canada, il s'agirait de la $5^{\mathrm{e}}$ zone urbaine fonctionnelle en importance au Québec et de la 26e au Canada. Malgré son poids démographique non négligeable, Saguenay et son voisinage immédiat forment une ville moyenne plutôt modeste si on la compare à la majorité des RMR canadiennes. À l'instar de régions métropolitaines telles que Sudbury, Thunder Bay ou Saint-John, Saguenay est localisée en périphérie des grands corridors urbains et industriels et sa population stagne ou décline au gré des recensements.

Ce phénomène de déclin relatif est associé à son économie peu diversifiée s'appuyant essentiellement sur les ressources naturelles ainsi qu'à sa difficulté à attirer des immigrants. En effet, Saguenay est la région métropolitaine la plus homogène au Canada avec plus de $98 \%$ de francophones. La présence de grandes entreprises anglo-saxonnes (Price brothers et Alcoa), au cours du XIX ${ }^{\text {e }}$ et
$\mathrm{XX}^{\mathrm{e}}$ siècles, de même que les grands chantiers industriels ou hydroélectriques ou la base militaire de Bagotville n’ont pas réussi à attirer et à maintenir à long terme des populations importantes d'origines diverses.

Dans ce contexte, notre article vise à dresser un portrait de l'agglomération de Saguenay, notamment sur les plans économique, démographique et urbanistique. Après une mise en exergue de son rôle de pôle régional, différentes interrogations seront abordées : quel est l'avenir des villes moyennes situées aux marges de l'écoumène, comme Saguenay? Comment accueillir des immigrants dans un environnement social très homogène? Faut-il appréhender un clivage progressif des besoins et des opportunités entre ces villes moyennes de l'arrièrepays et les grandes métropoles telles que Montréal? Nous tenterons de répondre à ces interrogations à l'aide d'informations documentaires et de quelques traitements statistiques et cartographiques.

a Professeur, membre du Centre de recherche sur le développement territorial (CRDT), Université du Québec à Chicoutimi 


\section{Saguenay : un pôle régional et une agglomération polycentrique}

Saguenay est le pôle incontesté de la région administrative du Saguenay-Lac-Saint-Jean. En fait, il s'agit de la seule agglomération québécoise de plus de 100000 habitants localisée en-dehors de la vallée du Saint-Laurent. Ainsi, plus de $50 \%$ de la population régionale habite à Saguenay (voir la figure 1). Si on utilise les limites de la région métropolitaine de recensement (RMR) définies par Statistique Canada en 2016, c'est près de $60 \%$ des Sagamiens $^{1}$ qui se retrouvent intégrés à l'unité socioéconomique de Saguenay ${ }^{2}$.

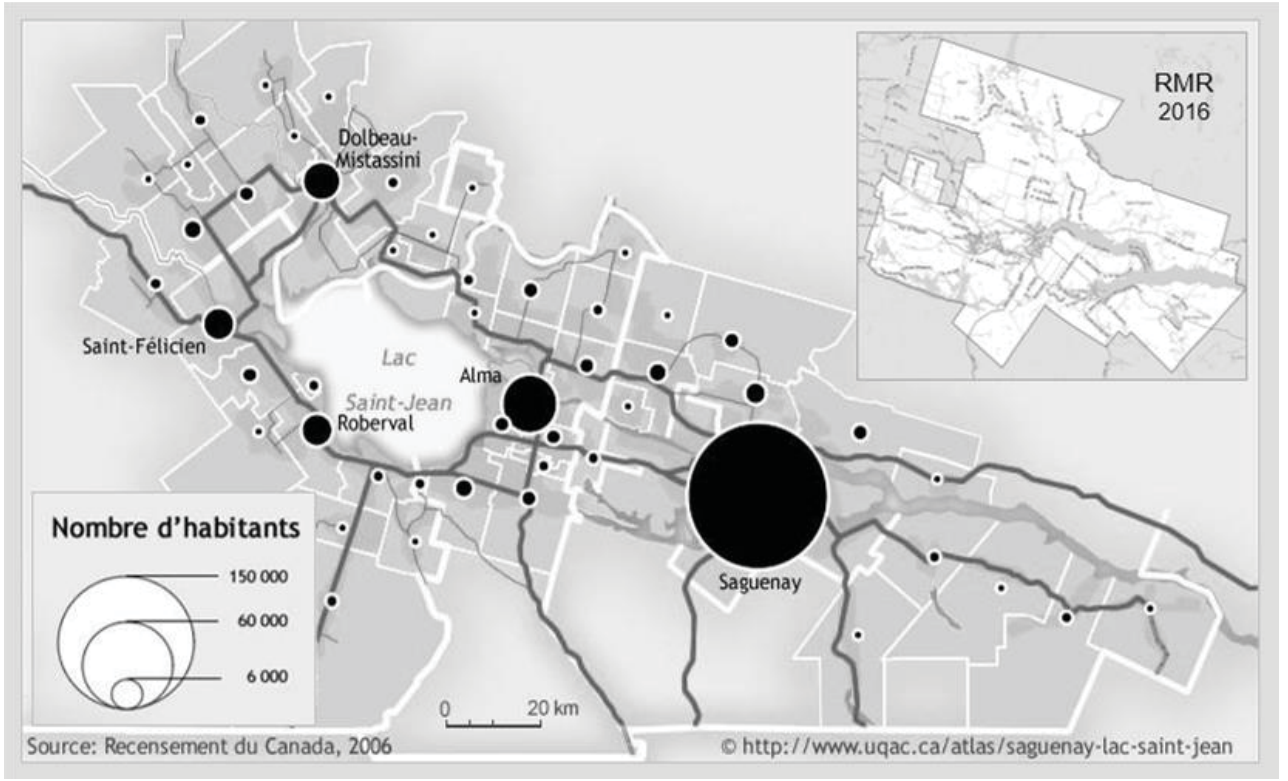

Figure 1-Saguenay comme pôle régional et RMR

Sources : Atlas électronique du Saguenay-Lac-St-Jean, 2018a et Statistique Canada, 2017

Ville industrielle et centre important de services publics et commerciaux, Saguenay paraît plutôt active et prospère aux yeux des habitants de municipalités rurales souvent dévitalisées situées à relative proximité. Elle vit néanmoins des difficultés structurelles qui soulèvent des inquiétudes pour l'avenir. D'une part, la modernisation des usines a fait diminuer le nombre d'emplois industriels. D'autre part, la mobilisation en faveur d'initiatives économiques innovantes est également laborieuse, les élites politiques locales ayant longtemps favorisé le statu quo, c'est-à-dire la prospection de grands projets industriels plutôt le développement de PME ou la mise en place de filières à fortes composantes technologiques (Proulx, 2007). Nous reviendrons à cet aspect ultérieurement.

En matière d'organisation spatiale, Saguenay a toujours été très éclatée. Pour une large part, cela provient de son histoire et de son cadre physique (relief et hydrographie). Dès les années 1950, on parle de conurbation pour décrire les villes jumelles de Chicoutimi et Jonquière (Cimon, 1958). Pour sa part, le géographe Louis-Marie Bouchard décrit le Haut-Saguenay comme ceci, dans les années 1970 :

«... [un ensemble] de villes autonomes ayant eu une origine différente mais qui, à un moment donné, ont accentué leurs relations, ont établi des liens étroits d'interdépendance et sont parvenues aujourd'hui à constituer un tout organique qui n'empêche pas chacune d'elles de garder son individualité » (1973, p. 21).

Cette urbanité diffuse entrecoupée d'espaces agricoles, qui s'est accrue depuis cette époque, est fortement marquée par l'étalement urbain qui ceinture les noyaux historiques de l'agglomération et «colonise » les espaces ruraux environnants. Il est ardu de s'y déplacer autrement qu'en voiture nonobstant l'existence de transports en commun. De plus, il n'y a pas véritablement de centre des affaires $^{3}$. Les centres-villes de Chicoutimi et de Jonquière étant d'envergure plutôt modeste, tout en étant en compétition avec les artères commerciales périurbaines, en particulier le boulevard Talbot (voir la figure 2). 


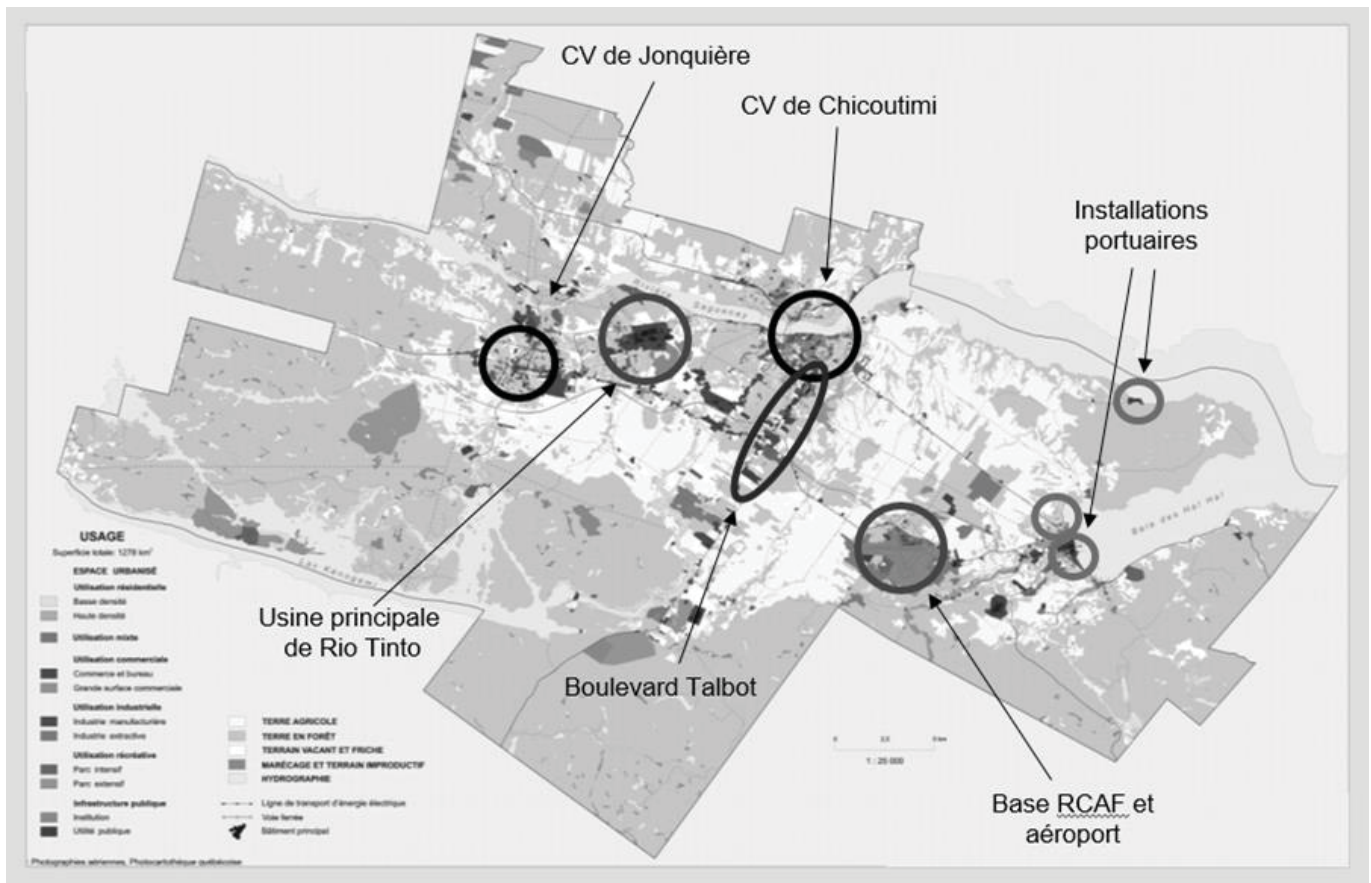

Figure 2 - Utilisation du sol, secteurs fonctionnels et grands équipements

Source : Atlas électronique du Saguenay-Lac-St-Jean, 2018b - ajouts de M. Simard

En conséquence, faire de cette zone urbaine polycentrique une ville durable constitue un défi de taille pour les urbanistes. En outre, les citoyens adhèrent en masse à l'idéal combiné de l'habitat pavillonnaire, de la camionnette et de la consommation ostentatoire. On peut parler de royaume du bungalow avec $75 \%$ des ménages vivant dans un logement individuel (Société canadienne d'hypothèques et de logement - SCHL, 2018). Par ailleurs, les terres à construire sont disponibles à distance raisonnable et à faible coûts, ce qui rend l'idée d'une ville plus compacte façonnée pour la marche ou le vélo des plus saugrenues pour la majorité des citoyens ${ }^{4}$.

Sur le plan administratif5, la Ville de Saguenay est issue de deux phases distinctes de regroupements municipaux qui ont progressivement réunies une vingtaine d'entités municipales en une seule (Simard et Goulet, 2005). La première phase a eu lieu en 1975 et 1976. Trois principales municipalités ont alors émergé : Jonquière (Jonquière, Arvida, Kénogami et Saint-Dominique-de-Jonquière), Chicoutimi (Chicoutimi, la paroisse de Chicoutimi, Chicoutimi-Nord et Rivière-du-Moulin) et La Baie (Bagotville, Paroisse de Bagotville, Grande-Baie et Port-Alfred). La deuxième phase a eu lieu en 2001 et 2002, le gouvernement du Québec adopte à ce moment le décret (841-2001) qui crée officiellement la Ville de Saguenay (Chicoutimi, Jonquière, La Baie, Canton-Tremblay ${ }^{6}$, Lac-Kénogami, Laterrière et Shipshaw) ${ }^{7}$.

Incidemment, la Ville de Saguenay est divisée en trois arrondissements portant le nom des trois principales Villes fusionnées, mais englobant les anciennes municipalités rurales adjacentes. Toutefois, ces arrondissements ont moins de pouvoir qu'à Québec et, surtout, qu’à Montréal. Saguenay dispose également de certaines compétences des Municipalités régionales de comté (MRC), comme 13 autres villes regroupées au Québec. Depuis 2002, la MRC du Fjord-du-Saguenay se retrouve à former une espèce de beigne composée de milieux ruraux entourant la Ville de Saguenay, sur les deux rives du fjord. Le siège administratif de la MRC est maintenant situé à Saint-Honoré. Celle-ci tire notamment des revenus de ses vastes territoires non-organisés ${ }^{8}$.

En plus de sa forme polycentrique et étalée, Saguenay présente trois particularités urbanistiques, selon nous. Tout d'abord, on remarque un site géographique riche et varié caractérisé par les plans d'eau, les dénivellations et les réseaux de ravins. Ce site est soumis aux risques naturels comme le démontre le 
glissement de terrain meurtrier de Saint-Jean-Vianney, en 1971, et les impressionnantes inondations de 1996, communément qualifiées de déluge! En deuxième lieu, Saguenay se démarque par la présence sur son territoire de trois anciennes villes de compagnie planifiée selon des plans urbains originaux (voir la figure 3), c'est-à-dire Kénogami (1912), Port-Alfred (1916) et Arvida (1926).

Cette dernière, qui est la plus imposante, s'inspire du courant esthétique et hygiéniste appelé City beautiful (Morisset, 1998). Il s'agit depuis peu d'un site patrimonial inscrit au répertoire du patrimoine culturel du Québec, le seul site de ce type dans la région du Saguenay - Lac-Saint-Jean (Pedneault, 2018) ${ }^{9}$. Troisièmement, de nombreux bâtiments d'architecture moderne ont été érigés à Saguenay, que ce soit des églises, des édifices institutionnels ou des résidences individuelles. Cela découle notamment de l'influence des célèbres architectes québécois Paul-Marie Côté et Léonce Desgagné, lesquels ont exercé leur art dans les années 1950 et 1960 (Morisset, 2004).

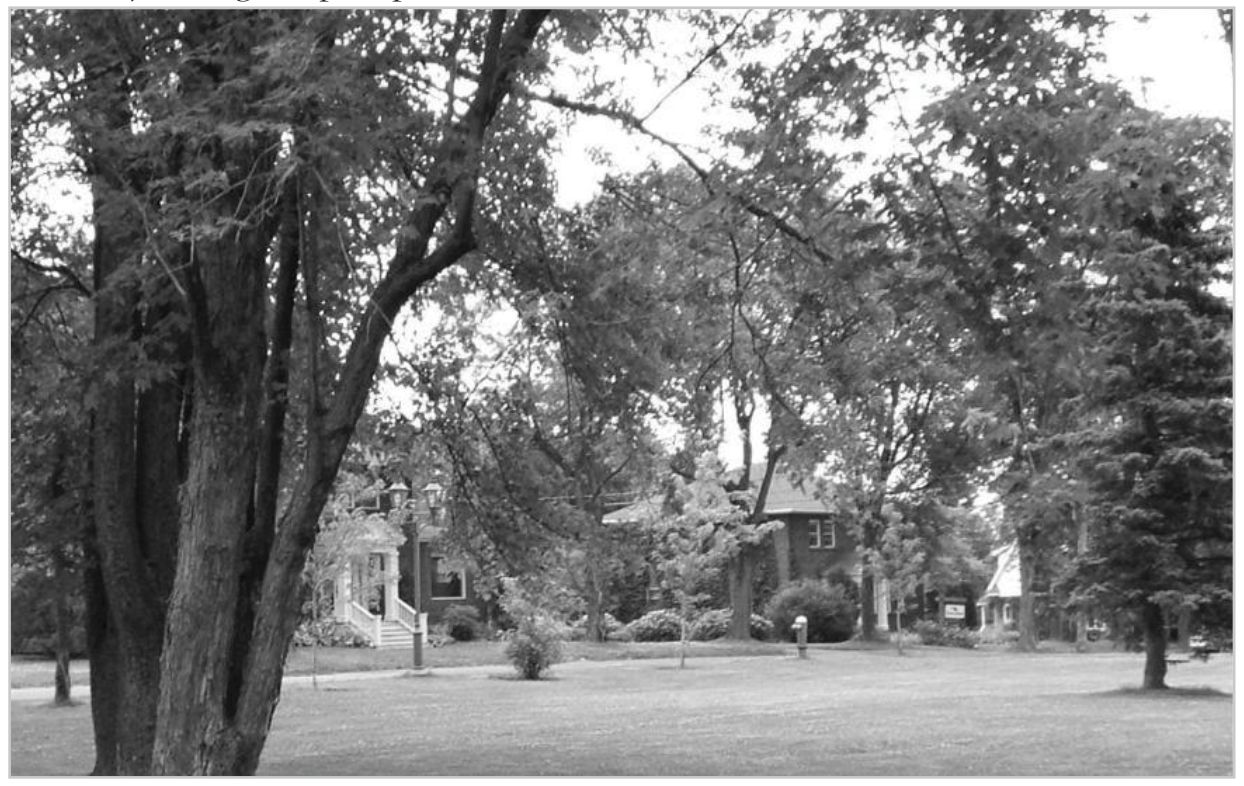

Figure 3 - «Quartier des Anglais » à Kénogami (Arrondissement de Jonquière) Source : Martin Simard

\section{Les tendances économique, démographiques et urbanistiques}

Tel que mentionné précédemment, la base économique de Saguenay et de la région du SaguenayłacSaint-Jean est associée aux ressources naturelles, notamment à la production d'aluminium et à la forêt. L'industrie forestière subit une crise plus ou moins chronique depuis environ une vingtaine d'années. Par exemple, la fermeture en 2003 de l'usine de papiers de l'Abitibi-Consolidated à La Baie a éliminé près de 700 emplois (voir la figure 4). De son côté, l'industrie de l'aluminium reste assez « robuste » avec des complexes industriels dans chacun des arrondissements. Cependant, le nombre d'emploi a été réduit de moitié depuis 30 ans.

En effet, ce processus peu visible se réalise par attrition avec en toile de fond le développement des technologies et l'automatisation de la production. Proulx souligne ce phénomène en parlant de paradoxe :

« [la] production d'aluminium primaire a doublé (de 450000 à plus d'un million de tonnes/année) alors que l'emploi direct associé a diminué de $66 \%$, soit de 9270 emplois en 1980 à autour de 3100 emplois en 2015 » (2017).

S'agit-il d'une économie d'une autre époque à l'heure de l'innovation, des technologies et de l'économie du savoir? Quelle que soit la réponse à cette question, les efforts de $2^{\text {e }}$ et $3^{\text {e }}$ transformations de l'aluminium et de diversification de l'économie donnent peu de résultats, jusqu'à maintenant. L'université, le développement commercial et les services de santé offrent des emplois intéressants 
mais le rêve des grands projets industriels demeure vivace malgré la montée de voix discordantes préoccupées par l'environnement (Simard et Blackburn, 2018). Actuellement, des projets miniers et gaziers sont à l'étude, ce qui occasionnerait une expansion des installations portuaires de GrandeAnse et un nombre significatif de nouveaux emplois (Munger, 2019).

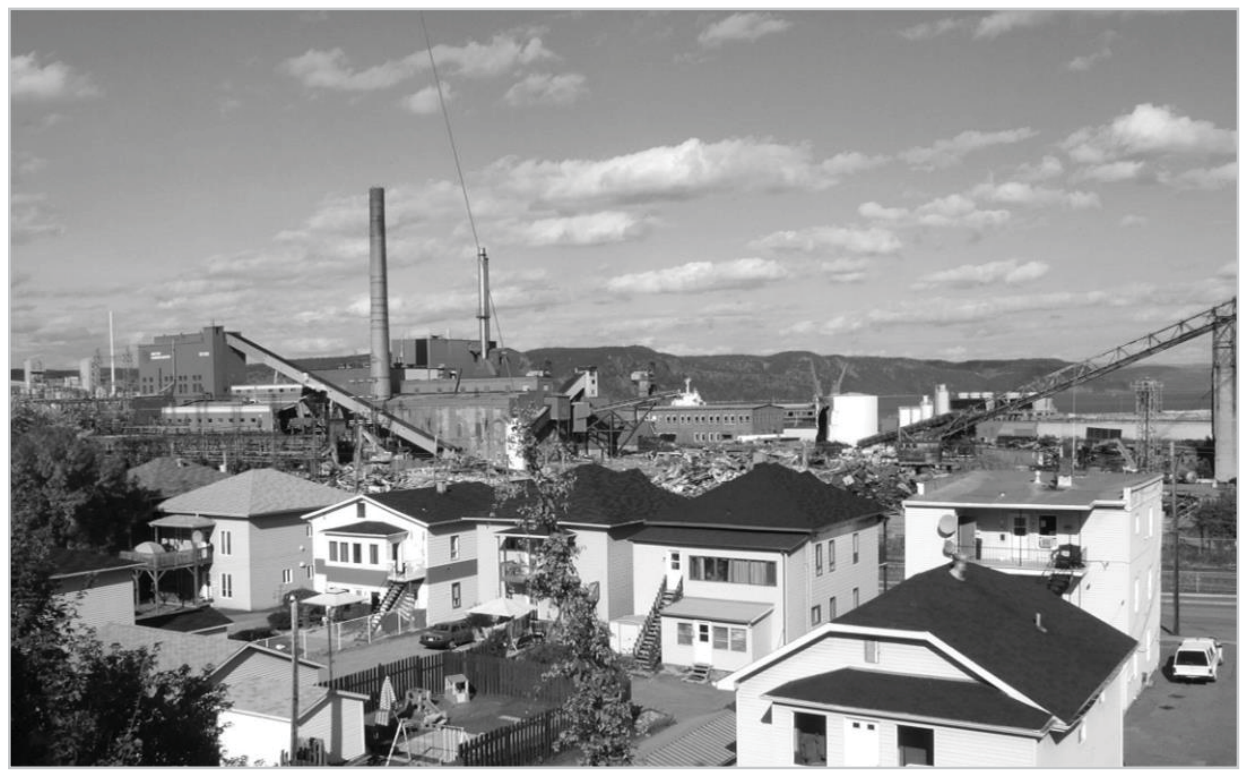

Figure 4 - Démolition de l'usine de l'Abitibi-Consolidated en 2006 (Arrondissement de La Baie) Source : Martin Simard

Ce contexte économique plutôt stagnant a eu des répercussions sur la démographie ${ }^{10}$. La figure 5 montre que les populations de la Ville et de la RMR de Saguenay ont décliné entre 1991 et 2016, quoique dans une proportion moindre que la décroissance enregistrée à l'échelle de la région administrative pour la même période ${ }^{11}$. Une analyse des recensements sur une base quinquennale illustre que des baisses importantes ont été observées dans les années 1990, mais que la situation s'est redressée à partir de 2006. Au final, les populations de la Ville et de la RMR demeurent légèrement plus basses en 2016 qu'en 1991, malgré la hausse de 1,5\% de la population de la RMR entre 2011 et 2016.

\begin{tabular}{|c|c|c|c|}
\hline & Population 1991 & Population 2001 & Population 2016 \\
\hline Saguenay (Ville) $^{1}$ & 151197 & 145172 & 145949 \\
\hline Saguenay (RMR) $^{2}$ & 162889 & 157233 & 160980 \\
\hline
\end{tabular}

Figure 5 - Évolution des populations de la Ville et de la RMR de Saguenay entre 1991 et 2016 Source : Statistique Canada, 1992, 2002 et 2017

La décroissance démographique est largement attribuable au phénomène de la migration des jeunes. En effet, le solde migratoire négatif des diverses cohortes de jeunes a atteint des proportions considérables dans les années 1990 et au début des années 2000 dans les villes et régions périphériques du Québec (Gauthier et al., 2006). Le tableau 6 indique d'ailleurs que le pourcentage de jeunes de 15 à 34 ans est inférieur à Saguenay par rapport aux trois autres RMR de taille semblable au Québec, lesquelles sont localisées dans des régions centrales ou intermédiaires. Le tableau serait probablement plus sombre pour Saguenay n'eut été de la mobilisation des acteurs régionaux qui ont mis de l'avant la stratégie de marketing territorial nommée MigrAction (Stecq, 2016). 


\begin{tabular}{|l|c|c|c|c|c|}
\hline & $\begin{array}{c}\text { Population } \\
\mathbf{2 0 1 6}\end{array}$ & $\begin{array}{c}\text { Variation } \\
\mathbf{2 0 1 1 / 1 6}\end{array}$ & $\begin{array}{c}\text { Population } \\
\text { de 15-34 ans }\end{array}$ & $\begin{array}{c}\text { Densité 2016 } \\
\text { (hab. par km }{ }^{2} \text { ) }\end{array}$ & $\begin{array}{c}\text { \% ménages en } \\
\text { logement ind. } \mathbf{1 2}^{2}\end{array}$ \\
\hline Gatineau & 332057 & $\mathbf{4 , 4} \%$ & $24,9 \%$ & 106,2 & $70,7 \%$ \\
\hline Sherbrooke & 212105 & $4,9 \%$ & $25,1 \%$ & 145,3 & $58,7 \%$ \\
\hline Saguenay & $\mathbf{1 6 0 9 8 0}$ & $\mathbf{1 , 5} \%$ & $\mathbf{2 2 , 2} \%$ & $\mathbf{5 8 , 3}$ & $\mathbf{7 4 , 5} \%$ \\
\hline Trois-Rivières & 156042 & $2,8 \%$ & $22,9 \%$ & 150 & $63,8 \%$ \\
\hline
\end{tabular}

Figure 6 - Comparaison de quatre RMR du Québec (populations, densités et logements) Source : Statistique Canada, 2012 et 2017

Les autres indicateurs de la Figure 6 expriment la position plutôt défavorable de Saguenay dans le club des villes moyennes supérieures québécoises. En outre, les contextes économique et démographique relativement moroses de la RMR de Saguenay auraient aussi des impacts défavorables sur l'industrie de la construction et le marché immobilier (voir la figure 7). Ainsi, les mises en chantier de type résidentiel suivent une courbe descendante depuis 2012 et les prévisions ne laissent pas présager d'amélioration à court terme. Également, le taux d'inoccupation des logements est assez élevé, à 6,8 \% en 2017, soit plus que les trois autres RMR comparées précédemment (SCHL, 2018). La construction de nouveaux secteurs domiciliaires risque d'être rare dans les prochaines années.

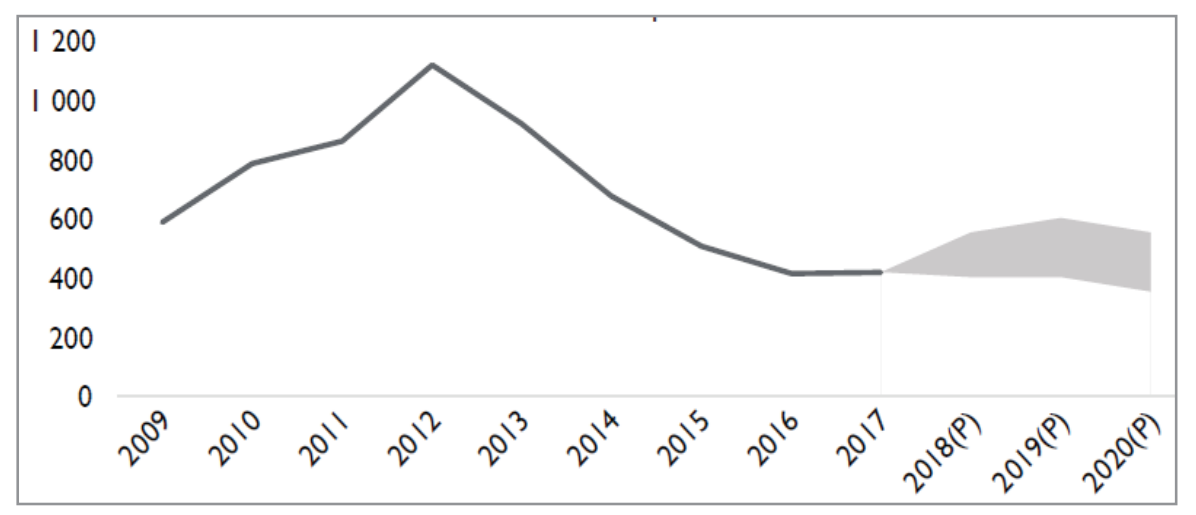

Figure 7 - Mises en chantier dans les RMR de Saguenay de 2009-2020 Source : donnés réelles et prévisions - SCHL, 2018

Fermeture d'usines, tissu urbain éclaté de faible densité, décroissance démographique, faible proportion de jeunes, tous ces éléments ont nourri la thèse du déclin de Saguenay selon le concept de Shrinking City (Tremblay, Meloche, et Scherrer, 2016). Cette expression décrit les villes en crises économique et démographique qui se retrouvent avec un espace urbain trop vaste pour la population présente et des bâtiments ou quartiers abandonnés (Wolff, Fol, Roth, et Cunningham-Sabot, 2013). On remarque plusieurs exemples de cela en Europe (Lodz en Pologne) ou, plus rarement, en Amérique du Nord (Détroit dans le Michigan). Cette conception de Saguenay comme ville « rétrécissante » nous semble un peu extrême, mais elle se fonde tout de même sur des réalités et des tendances inquiétantes pour la capitale sagamienne.

\section{Les défis de la diversité et de l'urbanité}

Sans être dramatique, la situation globale de Saguenay n'est pas rose. Le développement économique de la région métropolitaine ne peut se réaliser sans un certain redressement démographique, et vice versa. Jusqu'à récemment, les marchés immobiliers et de la construction avaient maintenu une certaine vigueur en dépit des aléas de la démographie, mais cette période de grâce est terminée. En effet, une ville en décroissance peut se développer un certain temps car la création de ménages (jeunes couples en ménages, 
divorces, etc.) et, conséquemment, la demande en nouveaux logements se poursuivent malgré les pertes de population. Toutefois, cette phase transitoire ne peut se maintenir à long terme.

La faible fécondité et le solde migratoire déficitaire d'une ville ou d'une région ne peuvent être comblés que par l'attraction de migrants nationaux ou d'immigrants. À cet égard, Saguenay a beaucoup de chemin à faire pour offrir de nombreux postes de travail et, en particulier, améliorer son image. L'administration municipale conservatrice en place de 2002 à 2017, sous la tutelle du Maire Jean Tremblay, s'est caractérisée par son populisme autoritaire et son peu d'ouverture face aux immigrants (Simard et Bergeron, 2011). Le combat largement médiatisé de l'ancien maire en faveur du maintien de la prière aux séances du conseil municipal a contribué à l'émergence d'une image de ville rétrograde de l'arrière-pays, dans tout le Québec.

Des politiques municipales sur des thèmes comme la famille, le développement social, le développement durable ou l'immigration existent à Saguenay. Cependant, celles-ci paraissent moins attrayantes que celles de villes comparables comme Sherbrooke, selon l'analyse de contenu réalisée par Chantale Munger (2016). De plus, leurs énoncés vertueux de ces documents sont souvent en décalage avec les discours à l'emporte-pièce des élus locaux de Saguenay. De surcroît, on doit composer avec la présence de groupes réactionnaires d'extrême droite, comme la Meute, groupes également actifs ailleurs dans la province (Maltais, 2017).

Il reste donc beaucoup à faire pour attirer de nouvelles populations à Saguenay. La beauté des paysages naturels, la quiétude des villes moyennes et le faible coût de la vie réussiront-ils à compenser l'homogénéité ambiante et la fermeture apparente d'une certaine frange de la population? En outre, l'environnement urbain de type banlieue sans véritable urbanité n'est pas très propice à l'accueil «d'une classe créative» jeune et dynamique, pour reprendre le terme de Richard Florida (Darchen et Tremblay, 2008). On voit mal quel secteur de l'agglomération de Saguenay pourrait devenir un «quartier latin» dense et animé, soit un terreau fertile pour les échanges et l'innovation.

Peut-être que la partie ouest du centre-ville de Chicoutimi devrait assumer ce rôle, comme le propose l'organisme «Promotion Saguenay» (Savard, 2019). Ce secteur comporte des équipements culturels et le réseau de transport en commun y est bien implanté. De plus, les espaces commerciaux et le parc de la zone portuaire constituent des atouts. Cependant, le projet d'espace public de la Place du citoyen a été mal reçu malgré son esthétique contemporaine (voir la figure 8). L'équipement a pourtant été beaucoup moins onéreux que le Quai d'escale de La Baie, genre d'éléphant blanc de près de 60 millions $\$$ (Munger, 2013) qui a eu très peu de retombées en dépit de la rhétorique politicienne (Blackburn, 2018).

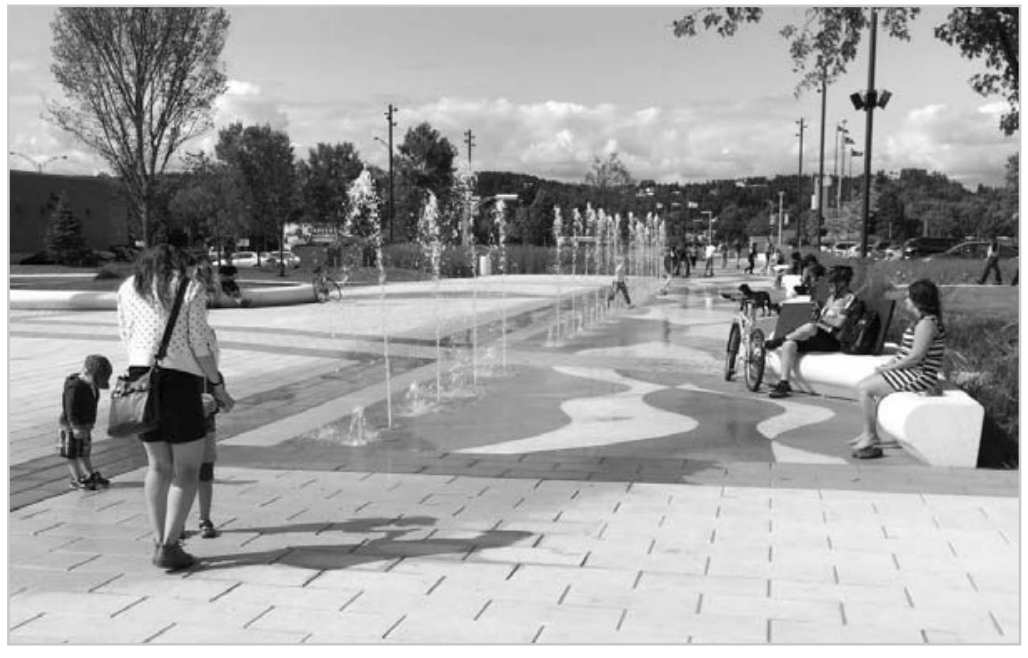

Figure 8 - La Place du citoyen - Arrondissement de Chicoutimi Source : Martin Simard 
La présence de l'entreprise informatique CGI et l'arrivée récente d'Ubisoft sur la rue Racine à Chicoutimi sont des signes positifs en faveur d'une diversité sociale et économique (Paradis, 2017). Par ailleurs, l'Université et les cégeps contribuent au cosmopolitisme naissant à travers l'inscription de bon nombre d'étudiants internationaux. Des politiques publiques innovantes et la créativité saguenéenne pourraient s'épanouir à partir de ces acquis, du moins on peut l'espérer. En 2017, l'arrivée d'une administration municipale plus «progressiste» est certainement de bon augure. Néanmoins, celle-ci fait face à un contexte financier contraignant et la fin de l'autoritarisme laisse rejaillir les tensions entre les arrondissements dans une ville qui ne s'est jamais appropriée son « nouveau » nom!

\section{Conclusion}

À l'instar de "petites régions métropolitaines » telles que Sudbury, Thunder Bay ou Saint-John, lesquelles sont localisées en périphérie des grands corridors urbains et industriels, Saguenay connaît des problèmes économiques et démographiques et est caractérisée par son homogénéité ethnoculturelle. Ce type d'agglomération endogénétique, c'està-dire branchée sur l'arrière-pays plutôt que sur l'international, a de la difficulté à relever les défis du changement et de la diversité, en particulier à attirer des immigrants, au même titre que les petites villes et les espaces ruraux. Il s'agit d'un enjeu majeur en contexte de faible natalité. L'attraction de migrants nationaux ou d'immigrants internationaux est la seule voie de croissance possible face à l'improbable augmentation de la fécondité.

Cette situation promet d'engendrer de multiples conséquences sur le marché de l'emploi, le développement urbain et immobilier, la croissance commerciale et le maintien de services publics de pointe. Alors que leur population stagne ou décline au gré des recensements, ces territoires s'isolent de plus en plus du nouvel univers mondialisé qui se déploie dans les grandes villes et les métropoles. Dans un monde de réseaux et de mobilité, cette subordination sociospatiale peut occasionner des effets de rétroaction rapides si des mesures correctives vigoureuses ne sont pas mises en œuvre pour rejoindre pleinement la société mondialisée au cours de la prochaine décennie.

La "prophétie» de Bourne et Simmons, énoncée en 2003, sur le clivage à venir entre les grandes métropoles multiculturelles, et les petites villes et milieux ruraux, paraît en voie de se concrétiser. Estce que Saguenay saura faire mentir cette prédiction peu enthousiasmante en ce qui a trait à l'aménagement, au développement et à la cohésion sociale? La balle est dans le camp des politiciens, des gens d'affaires et de l'ensemble des citoyens! Pour l'instant, il semble que les débats plus triviaux, comme celui sur le montant des taxes municipales, canalisent les énergies de la population et des intervenants locaux.

\section{NOTES}

1 Gentilé occasionnellement utilisé par décrire les habitants de la «Sagamie », terme synonyme de Saguenay-Lac-Saint-Jean.

2 Plus précisément, la RMR de Saguenay comptait, en 2016, 160980 habitants répartis dans 9 municipalités, soit un territoire de $2760 \mathrm{~km}^{2}$ (densité de population de 58,3 h./km2). Les villes et villages limitrophes à Saguenay sont considérés comme faisant partie de la RMR dans la mesure ou plus de $50 \%$ de la population active de ceux-ci travaille sur le territoire de la Ville de Saguenay (Bégin, Larouche, Saguenay, Saint-Charles-de-Bourget, Saint-David-de-Falardeau, Sainte-Rose-du-Nord, Saint-Félix-d'Otis, SaintFulgence et Saint-Honoré). Pour plus de détails voir le site Internet https://www12.statcan.gc.ca/census-recensement/2016/ as-sa/fogs-spg/Facts-cma-fra.cfm?LANG=Fra\&GK=CMA\&GC=408\&TOPIC=1.

3 Central Business District ou CBD, en anglais.

4 La Ville de Saguenay possède malgré tout un Guide de développement durable encourageant les bonnes pratiques dans une perspective de durabilité faible (récupération, recyclage des déchets, limiter l'usage des pesticides, etc.).

5 Soulignons ici que la RMR sert à compiler des statistiques sur la base du bassin d'emploi, espace qui correspond aussi à l'aire immédiate de rayonnement des services. Il ne s'agit pas d'une instance politique ou administrative.

6 En fait, le Canton-Tremblay a été scindé en deux. Une partie a été réunie à Saguenay et l'autre à la municipalité de Saint-Honoré.

7 La nouvelle Ville entre en opération le 18 février 2002, le maire et les conseillers ayant été élus en novembre 2001.

8 Redevances provenant des barrages privés, revenus liés à la délégation de la gestion par le Ministère de l'Énergie et des ressources naturelles des baux de villégiature sur les terres publiques, etc.). 
9 Repéré à http://www.patrimoineculturel.gouv.qc.ca/rpcq/detail.do?methode= consulter\&id $=168589$ \&type $=$ bien $\#$. XDYroFxKjIU

10 On remarque une amélioration du marché de l'emploi à Saguenay depuis quelques années.

11 Selon les données de l'Institut de la statistique du Québec, la population du Saguenay-Lac-Saint-Jean est passée de 286 159, en 1991, à 276 509, en 2017, soit une baisse de 3,4\%.

12 Regroupement des maisons individuelles attenantes, non-attenantes, jumelées, en rangée, duplex maisons mobiles divisé par le \% de ménages privés en 2016.

\section{RÉFÉRENCES}

Blackburn, R. (2018, 18 juillet). Les croisières à Saguenay. 1,5 million de dollars par année depuis dix ans, Le Quotidien. Repéré à https://www.lequotidien.com/actualites/15-m--par-annee-depuis-10-ans-9c73dc32c6de4b17c784b2168d45fc23.

Bouchard, L.-M. (1973). Les villes du Saguenay : étude géographique. Montmagny, QC: Leméac et FUQAC.

Bourne, L. S. et Simmons, J. (2003). New fault lines? Recent trends in the canadian urban system and their implications for planning and public policy. Canadian Journal of Urban Research / Revue canadienne de recherche urbaine, 12(1), 22-47.

Cimon, J. (1958). Un exemple canadien de planification : la conurbation du Haut-Saguenay. Cabiers de géographie du Québec, 3(5), 43-46.

Darchen, S. et Tremblay, D.-G. (2008). La thèse de la « classe créative » : son incidence sur l'analyse des facteurs d'attraction et de la compétitivité urbaine. Interventions économiques, 37. Repéré à https://journals.openedition.org/ interventionseconomiques/503.

Fontaine, J. (2017, Octobre). Bilan et perspective du marché immobilier résidentiel. Communication présentée à Xplor 2017, Montréal, QC. Repéré à https://www.aemq.qc.ca/documentation/conferences/2017/2017-06_Congres5_ Perspectives_marche_immo_residentiel.pdf

Gauthier, M., Leblanc, P., Côté, S., Deschenaux, F., Girard, C., Laflamme, C., ... Molgat, M. (2006). La migration des jeunes an Québec. Rapport national d'un sondage 2004-2005 auprès des 20-34 ans du Québec. Montréal, QC: Institut national de la recherche scientifique, Urbanisation, culture et société, Observatoire jeunes et société.

Gauthier, M. et al. (2018a). Atlas électronique du Saguenay-Lac-Saint-Jean. Repéré à http://atlas.uqac.ca/saguenay-lac-saintjean $/$ ?P $=$ Liste $\% 20 \mathrm{des} \% 20$ cartes\&S=3_2_1_3\&L=fr\#3_2_1_3

Gauthier, M. et al. (2018b). Atlas électronique du Saguenay-Lac-Saint-Jean. Repéré à http:/ / atlas.uqac.ca/saguenay-lac-saintjean $/$ ?P $=$ Liste $\% 20 \mathrm{des} \% 20$ cartes\&S=3_2_8_1\&L=fr\#3_2_8_1

Morisset, L. K., Noppen, L. et Dieudonné, P. (2004). Patrimoines modernes : l'architecture du vingtième siècle à Chicoutimi. Québec, QC: Presses de l’Université du Québec.

Morisset, L. K. (1998). Arvida, cité industrielle. Une épopée urbaine en Amérique. Québec, QC: Septentrion.

Maltais, P.-A. (2017, 20 juillet). Une pancarte Saguenay ville blanche retirée du cimetière. Journal de Québec. Repéré à https://www.journaldemontreal.com/2017/07/20/saguenay-ville-blanche-une-affiche-a-connotation-raciste-a-lentreedun-cimetiere.

Munger, C. (2016). Comparaison des politiques municipales de Saguenay et Sherbrooke en matière d'attractivité urbaine. Organisations et territoires, 25(1), 5-82. Repéré à https://doi.org/10.1522/revueot.v25i1.71

Munger, G. (2019, 2 janvier). Les grands projets industriels à surveiller en 2019 au Saguenay-Lac-Saint-Jean. Société RadioCanada. Repéré à https://ici.radio-canada.ca/nouvelle/1144759/les-grands-projets-industriels-a-surveiller-en-2019-ausaguenay-lac-saint-jean.

Munger, G. (2013, 9 juillet). 57 M \$ pour les croisières à La Baie. Société Radio-Canada. Repéré à https://ici.radiocanada.ca/nouvelle/622420/village-portuaire-la-baie.

Paradis, C. (2017, 5 septembre). Ubisoft confirme la création de 125 emplois à Saguenay. Société Radio-Canada. Repéré à https://ici.radio-canada.ca/nouvelle/1053955/ubisoft-creation-100-emplois-saguenay.

Pedneault, S. (2018, 25 novembre). Arvida reconnu par Québec comme premier site patrimonial dans la région. Société Radio-Canada. Repéré à https://ici.radio-canada.ca/nouvelle/1137973/site-patrimonial-valeur-preservation-gouvernement-saguenay. 
Proulx, M.-U. (2017, 9 février). Rapport aluminium 2016 [Billet de blogue]. Repéré à http://muproulx.com/bloguedeveloppement/e1-rapport-aluminium-2016

Proulx, M.-U. (2007). Vision 2025. Le Saguenay-Lac-Saint-Jean face à son avenir. Québec, QC: Presses de l’Université du Québec.

Société canadienne d'hypothèques et de logement (SCHL). (2018). Perspectives du marché de l'babitation. Région du Québec. Repéré à https://www.cmhc-schl.gc.ca/fr/data-and-research/publications-and-reports/housing-market-outlook-highlights.

Savard, D. (2019, 19 janvier). Promotion Saguenay veut un quartier numérique sur la rue Racine. Informe Affaires.com. Repéré à https://informeaffaires.com/regional/technologie/promotion-saguenay-veut-un-quartier-numerique-sur-la-rue-racine.

Simard, M. et Bergeron, G. (2011). L'administration du maire Tremblay à Saguenay ou le populisme fiscal à l'épreuve de la gouvernance. Dans S. Breux, L. Béhrer et J.-P. Collin (dir.). Portraits de la démocratie urbaine an Québec : les élections municipales de 2009. (pp. 209-232). Québec, QC: Presses de l’Université Laval.

Simard, M. et Goulet, A. (2005). La question identitaire lors des débats sur les regroupements municipaux de 1975-1976 au Saguenay. Saguenayensia, 47(1), 143-149.

Simard, R. et Blackburn, J. (2018, 10 novembre). Près de 300 manifestants dénoncent les projets industriels dans le fjord du Saguenay. Société Radio-Canada. Repéré à https://ici.radio-canada.ca/nouvelle/1135171/manifestation-fjord-ariannephosphat-black-rock-coalition-protection.

Statistique Canada (2017). Recensement de 2016 - Série Perspective géographique - RMR de Saguenay. Repéré à https://www12.statcan.gc.ca/census-recensement/2016/as-sa/fogs-spg/Facts-cmafra.cfm?LANG $=$ Fra\&GK=CMA\&GC $=408 \&$ TOPIC $=1$.

Statistique Canada (2012). Recensement de 2011 - Série Perspective géographique - RMR de Saguenay. Repéré à https://www12.statcan.gc.ca/census-recensement/2011/as-sa/fogs-spg/Facts-cmafra.cfm?LANG=Fra\&GK=CMA\&GC $=408$.

Stecq, H. (2016). La mobilisation des acteurs régionaux face à la migration des jeunes : le cas de la Stratégie MigrAction au Saguenay-Lac-St-Jean (Québec). Organisations et territoires, 25(1), 69-74. Repéré à https://doi.org/10.1522/revueot.v25i1.70

Tremblay, M., Meloche, J.-P. et Scherrer, F. (2016). Décroissance démographique et planification du territoire à Saguenay. Revue canadienne des sciences régionales / Canadian Journal of Regional Science, 39(1), 51-60.

Wolff, M., Fol, S., Roth, H. et Cunningham-Sabot, E. (2013). Shrinking Cities, villes en décroissance : une mesure du phénomène en France. Cybergeo : Revue européenne de géographie, 661. Repéré à https://doi.org/10.4000/cybergeo.26136 